\title{
Calculated Momentum Dependence of Zhang-Rice States in Transition Metal Oxides
}

\author{
Quan Yin ${ }^{1}$, Alexey Gordienko ${ }^{1,2}$, Xiangang Wan ${ }^{1,3}$, Sergey Y. Savrasov ${ }^{1}$ \\ ${ }^{1}$ Department of Physics, University of California, Davis, CA 95616 \\ ${ }^{2}$ Department of Physics, Kemerovo State University, Kemerovo, Russia and \\ ${ }^{3}$ National Laboratory of Solid State Microstructures and Department of Physics, Nanjing University, Nanjing, China
}

(Dated: April 23, 2022)

\begin{abstract}
Using a combination of local density functional theory and cluster exact diagonalization based dynamical mean field theory, we calculate many body electronic structures of several Mott insulating oxides including undoped high $\mathrm{T}_{c}$ materials. The dispersions of the lowest occupied electronic states are associated with the Zhang-Rice singlets in cuprates and with doublets, triplets, quadruplets and quintets in more general cases. Our results agree with angle resolved photoemission experiments including the decrease of the spectral weight of the Zhang-Rice band as it approaches $\mathbf{k}=0$.
\end{abstract}

Quasiparticle excitations in insulating transition metal oxides (TMOs) such as classical Mott-Hubbard systems or undoped high temperature superconductors (HTSCs) have been puzzling electronic structure theorists for many years 1, 2]. While photoemission experiments in these materials show [3] the existence of the $d$-states located both right below the Fermi energy and at much higher binding energies (typically $\sim 10 \mathrm{eV}$ ), it is difficult to understand this genuine many-body redistribution of the spectral weight using calculations based on a static mean field theory [4, 5], such as the density functional theory (DFT) in its local density approximation (LDA) [6]. Modern approaches, such as LDA $+\mathrm{U}$ 7], can differentiate between charge-transfer and Mott-Hubbard natures of these systems [8], but still have difficulties in recovering insulating behavior of the paramagnetic (PM) state and tackling more complicated many-body features such as Zhang-Rice (ZR) singlet of HTSCs [3, 9]. Only most recent developments based on a combination of local density approximation (LDA) and dynamical mean field theory (DMFT) [10] have started to address those issues [11, 12, 13].

In the present work, using a novel implementation of LDA plus cluster exact diagonalization based DMFT we demonstrate how to obtain accurate spectra of transition metal oxides and, in particular, describe full momentum dependent low-energy excitations associated in those systems with antiferromagnetic (AFM) Kondo-like coupling between a spin of oxygen hole injected by photoemission process and a local magnetic moment of the transition metal ion. These narrow energy bands are composed from the well known Zhang-Rice singlet states in cuprates [9], which have recently renewed their attention in connection with the disappearance of their spectral weight as the wave vector approaches the Brillouin Zone (BZ) center, and the observed high energy kink entitled as "waterfall" feature [14]. Zhang-Rice doublets have been discussed in $\mathrm{NiO}$ [15], and their further generalizations to triplets $(\mathrm{CoO})$, quadruplets $(\mathrm{FeO})$ and quintets $(\mathrm{MnO})$ all naturally emerge from our LDA+DMFT calculations. We find that the ZR states exhibit a similar behavior in all systems including the loss of their spectral weight at the $\Gamma$ point, which can be understood as the lack of hybridization between transition metal $d$ states and neighboring oxygen $p$ states, the effect most pronounced in HTSCs. There is a generally good agreement between our results and angle resolved photoemission (ARPES) experiments.

In our self-consistent LDA+DMFT calculations, the LDA one-electron Hamiltonians are supplemented with the self-energies of the $d$-electrons that are extracted from the cluster exact diagonalizations involving a $d$-shell of a transition metal ion hybridizing with nearest oxygen orbitals. We also include the effect of inter-site selfenergies in the study of cuprates including $\mathrm{Sr}_{2} \mathrm{CuO}_{2} \mathrm{Cl}_{2}$ and $\mathrm{La}_{2} \mathrm{CuO}_{4}$ by considering clusters of two copper $3 d$ orbitals. The hybridization functions in our calculations are fit using a single pole approximation $\Delta(\omega)=V^{2} /(\omega-$ $\left.E_{p}\right)$ which delivers the effective position of the bath level $E_{p}$ (interpreted as the oxygen $p$ band), as well as its hybridization $V$ with the $d$ states. The cluster self-energies are fit into a rational form $\Sigma(\omega)=\Sigma(\infty)+\sum_{i} W_{i} /\left(\omega-P_{i}\right)$ using interpolation by three poles [16]. Such fits allow to simplify the process of extracting parameters for the cluster Hamiltonians and performing full self-consistency using the LDA+DMFT for the actual materials considered in our work. Conventional values for the Coulomb interaction matrices, which were found from constrained density functional calculations [7], are used to perform the calculations at temperatures above the long-range magnetic order.

Since dimensions of the many-body Hamiltonians quickly become prohibitively large to handle by standard diagonalization algorithms, we have newly implemented a kernel polynomial method (KPM) for extracting spectral functions in our cluster calculations [17]. Similar to the Lanczos method, the KPM is an iterative procedure which allows to recover moments of many body densities of energy states. Using 200 moments or so, the oneelectron Green's functions are found to converge accurately for our cluster Hamiltonians with the dimensions up to 100,000 .

We first discuss a long-standing problem of low energy excitations in HTSCs. Early LDA calculations 

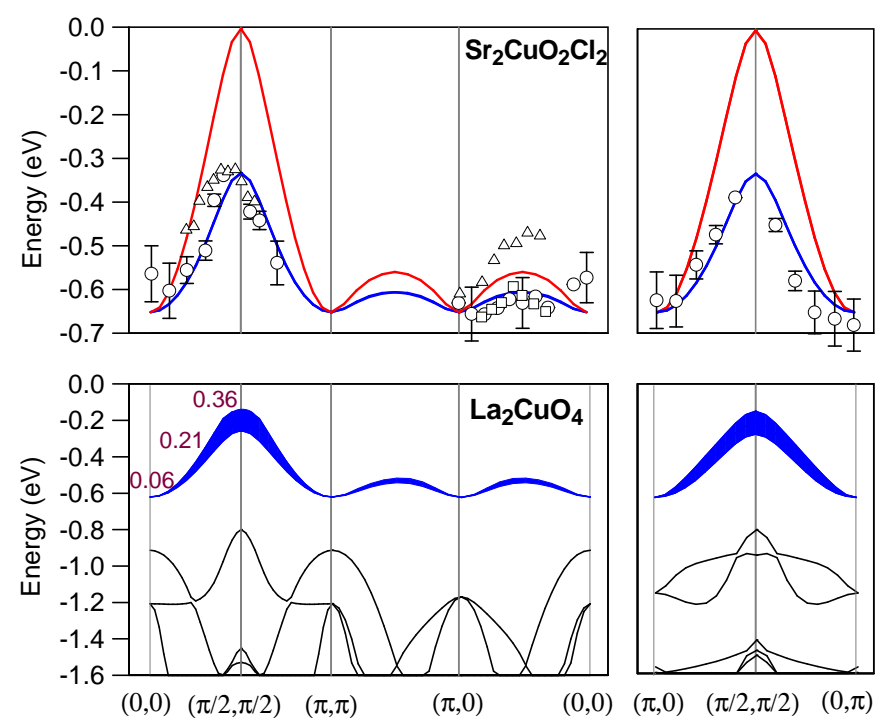

FIG. 1: (color online) Calculated low energy excitations in $\mathrm{Sr}_{2} \mathrm{CuO}_{2} \mathrm{Cl}_{2}$ (top) and $\mathrm{La}_{2} \mathrm{CuO}_{4}$ (bottom) using LDA plus single-site DMFT (red line) and two-site cluster DMFT (blue line). The experimental data are from: Ref. [19] (open circles), Ref. 20] (open triangles), Ref. 21] (open squares). The blue linewidth shows the oxygen content, while the numbers indicate the amount of electrons in the ZR band.

were unable to reproduce even the magnetic behavior of those systems [2]. Although this problem was later solved by the $\mathrm{LDA}+\mathrm{U}$ method 7], these calculations miss the important ZR physics and are unable to address the low-energy scale. On the other hand, $t-J$ and $t-t^{\prime}-t^{\prime \prime}-J$ model calculations have successfully reproduced the ZR dispersion as compared to the ARPES data [18, 19, 20, 21, 22]. A hot discussion has appeared in the literature around most recent ARPES experiments which have shown that the ZR band vanishes as the wave vector approaches the $\Gamma$ point, with the spectral weight seemingly transferring to higher-energies as the "waterfall" [14]. While it could be simple photoemission matrix element effect, most recent dynamical cluster approximation (DCA) based simulations of the 2D Hubbard model show 23] large imaginary part of the self-energy, resulting in asymmetric spectral functions resembling the waterfall. As we generally see, the HTSCs still represent a challenge for the theory, particularly for modern electronic structure calculations, which try to incorporate all hopping integrals accurately and use realistic values of the Coulomb interaction parameters.

In the present work LDA+DMFT calculations are performed for $\mathrm{Sr}_{2} \mathrm{CuO}_{2} \mathrm{Cl}_{2}$, whose ARPES experiments clearly identified the ZR band [3], and for $\mathrm{La}_{2} \mathrm{CuO}_{4}$. First, the calculations were done for a single impurity case when the cluster is made of a copper ion surrounded by 4 oxygen orbitals. This result is presented in Fig 1(top panel) by red line together with various photoemission experiments denoted by symbols. The dispersions of the ZR band in $\mathrm{Sr}_{2} \mathrm{CuO}_{2} \mathrm{Cl}_{2}$ correctly follows the ARPES data, although its bandwidth is overestimated by a factor of two, especially along $(0,0)-(\pi, \pi)$ direction. The situation is somewhat unclear along $(0,0)-(\pi, 0)$ line since experiments by two groups show quite different bandwidths (triangles 20] vs circles [19] and squares 21]). From the standpoint of the single-impurity model, it is however clear that the dispersion of the ZR band above the Néel temperature is primarily governed by the short-range antiferromagnetic correlations in that once the hole is moving in the lattice it is dressed up if the short-range order is present. This effect is missing when the singleimpurity approximation is adopted as the DMFT treats the PM regime as completely disordered state, resulting in the ZR band being too wide in our calculation. To include short range magnetic correlations, we subsequently performed calculations with two impurities by considering a cluster of two copper atoms surrounded by 7 oxygens (one shared orbital). The cluster exact diagonalization now delivers both the on-site $\Sigma_{11}(\omega)$ and inter-site $\Sigma_{12}(\omega)$ self-energies, which can be Fourier transformed to the form $\Sigma(\mathbf{k}, \omega)=\Sigma_{11}(\omega)+\Sigma_{12}(\omega)\left(\cos k_{x}+\cos k_{y}\right)$ and acquire the much needed $\mathrm{k}$-dependence. By fixing the frequency $\omega$ to the position of the ZR band we are now able to add $\Sigma(\mathbf{k}, \omega)$ to the LDA Hamiltonian. The result of such a calculation is depicted by the blue lines in Fig 1, A remarkable band narrowing now occurs where the $\mathrm{ZR}$ bandwidths come about $0.3 \mathrm{eV}$ for $\mathrm{Sr}_{2} \mathrm{CuO}_{2} \mathrm{Cl}_{2}$ and $0.4 \mathrm{eV}$ for $\mathrm{La}_{2} \mathrm{CuO}_{4}$. Using the calculated $\mathrm{ZR}$ dispersion, the parameters for $t-J$ model are also evaluated. For $\mathrm{Sr}_{2} \mathrm{CuO}_{2} \mathrm{Cl}_{2}$ they appear to be $J=0.135 \mathrm{eV}$, $t=0.34 \mathrm{eV}, t^{\prime}=-0.12 \mathrm{eV}$ and $t^{\prime \prime}=0.08 \mathrm{eV}$, and for $\mathrm{La}_{2} \mathrm{CuO}_{4}$ the numbers are $J=0.175 \mathrm{eV}, t=0.44 \mathrm{eV}$, $t^{\prime}=-0.15 \mathrm{eV}, t^{\prime \prime}=0.10 \mathrm{eV}$. The reduction of the $\mathrm{ZR}$ bandwidths by including $\mathrm{k}$-dependence of the self-energy demonstrates the importance of going beyond the singlesite approximation in describing the low-energy excitations in cuprates.

We further discuss the effect of disappearance of the ZR spectral weight around the $\Gamma$ point. In Fig 1, the blue fat line on the bottom panel shows the amount of oxygen $\mathrm{p}$ states presented in the $\mathrm{ZR}$ band of $\mathrm{La}_{2} \mathrm{CuO}_{4}$. It is clearly seen that the spectral weight gets smoothly reduced as the $\mathbf{k}$ vector approaches the BZ center. The same effect is found in our calculations for other HTSCs. The reduction can be simply understood using the 3-band tight-binding argument: the $\mathrm{k}$-dependent hybridization between $d_{x^{2}-y^{2}}$ and $p_{x}, p_{y}$ orbitals is exactly equal to zero at $\Gamma$, forbidding the formation of the ZR singlet. We can also track this effect with $\Sigma(\mathbf{k}, \omega)$ using a recently proposed matrix expansion algorithm [16]: Assuming that two poles of the self-energy are needed to reproduce both the Hubbard and the ZR bands, the resulting Hamiltonian is $5 \times 5$ in the extended pole space ( 3 for $d_{x^{2}-y^{2}}, p_{x}, p_{y}$ and 2 for the self-energy poles). It can 


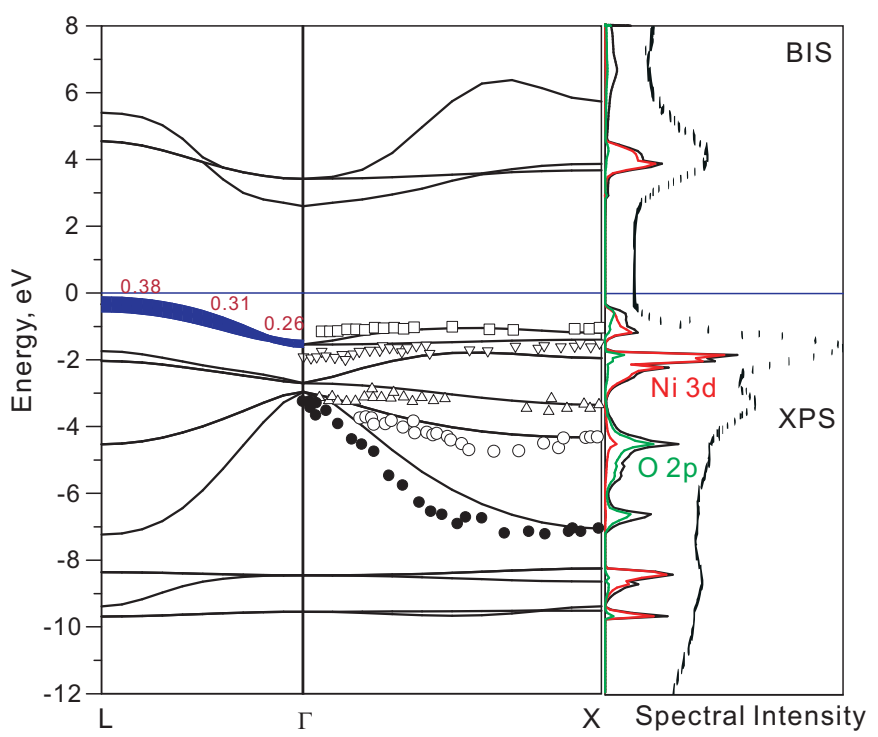

FIG. 2: (color online) Comparison between calculated quasiparticle dispersions using LDA+DMFT and the photoemission data [5, 25] for paramagnetic state of $\mathrm{NiO}$. The blue linewidth and the numbers show the oxygen content and the amount of electrons in the ZR band.

be simply diagonalized and the same effect is monitored. The amount of electrons in each band is less than one since auxiliary pole states are added to the Hamiltonian [16]. Fig[1 shows the actual number of electrons in the ZR band as a function of $\mathrm{k}$, decreasing towards $\Gamma$. Here, all spectral weight gets transferred to the Hubbard bands located at the scale of $U$ and also to the oxygen band.

While we are unable to account for the effect of the imaginary self-energy in the exact diagonalization for small clusters, recent DCA simulations of the Hubbard model [23] have shown very asymmetric $\mathrm{k}$-dependent spectral functions resembling the waterfall. On top of that a lot of other states are present below $-1 \mathrm{eV}$, as shown in Fig 1 (bottom panel). In our opinion this provides most natural interpretation of the recent ARPES data. As small hole doping occurs, the Fermi level sits at top of the ZR band which would again contradicts with the standard LDA like description of the cuprates, although we are unable to study the regime of optimal doping by the present procedure requiring too large clusters to diagonalize.

Let us now turn to the discussion of $\mathrm{NiO}$ which has been widely chosen as another classical example of a strongly correlated system. Two decades ago Fujimori et al 24] and Sawatzky et al [25] have shown that the valence band photoemission spectrum of $\mathrm{NiO}$ can be understood from a configurational interaction (CI) approach, indicating that the electronic properties here are local. The $3 d$ electrons repulsion gives rise to the lower and upper Hubbard bands located at -9 and $4 \mathrm{eV}$, respec- tively. The $3 d-2 p$ hybridization is responsible for the $3 d-$ character peak just below the Fermi level [26]. The discussion of a generalized spin-fermion model derived for a slab of $\mathrm{NiO}$ shows a well-developed set of ZR states which agree reasonably well within the low-energy features seen in the ARPES experiments [15]. However, $\mathrm{NiO}$ exhibits both correlation effects and band-structure effects, plus antiferromagnetic order under the Néel temperature of $520 \mathrm{~K}$. Below $\mathrm{T}_{N}$ extensive studies of $\mathrm{NiO}$ have been performed using the LDA $+\mathrm{U}$ method, and the positions of the Hubbard bands have been predicted correctly when using the values of on-site Hubbard interaction $U=8$ $\mathrm{eV}$ [7]. Unfortunately, much of the $d$-electron spectral weight just below the Fermi level is found to be lacking in this type of calculation unless the value of $U$ is reduced to $4 \sim 5 \mathrm{eV}$ making this system of Mott-Hubbard type rather than of charge-transfer type. Better than $\mathrm{LDA}+\mathrm{U}$, by using atomic (Hubbard I) approximation for the self-energy, one can get insulating solution even for paramagnetic case 11]. Also, recent comparisons between LDA calculations of $\mathrm{NiO}$ in its paramagnetic (predicted to be metallic) state and the ARPES experiments have indeed revealed [5] a rather good agreement between each other in the directions of BZ where the LDA band dispersions remain insulating. One thus faces a dilemma of matching different portions of the photoemission data with three different types of electronic structure calculations (LDA+U with two values of $\mathrm{U}$ as well as straight LDA).

We now show that all these problems can be overcome by performing LDA+DMFT calculations. Fig 2 shows the comparison between calculated quasiparticle dispersions and photoemission data for $\mathrm{NiO}[5,25]$. The $\mathrm{Ni} 3 d$ spectral weight is redistributed between the lower Hubbard band located at $-8 \sim-10 \mathrm{eV}$, upper Hubbard band located at around $4 \mathrm{eV}$, and strong peaks located just below the Fermi level. Having the ground state in $\mathrm{NiO}$ as $\left|p^{6} d^{8}\right\rangle$, the lowest excitation here is the result of the interaction between the spin of the oxygen hole and the two hole $S=1$ state in the $d$-shell. The total spin of such cluster can be $1 / 2$ (doublet) or $3 / 2$ (quadruplet). The antiparallel Kondo-like coupling implies that the doublet state is lower in energy, in accord with early predictions using the spin-fermion model [15]. The situation is akin to the singlet and triplet situation in cuprates. The direct energy gap is seen in Fig 2 to be around $3.5 \mathrm{eV}$ in our calculation (the experimental value is $4 \mathrm{eV}$ ), although most of the optical transitions would start at the energies $4 \mathrm{eV}$ or so as is evident from our density of states plots. We also see that both the positions and the dispersion of the oxygen bands located at the energies $-4 \sim-7 \mathrm{eV}$ below the Fermi level agree well with the ARPES data.

It is remarkable that similar to the HTSCs the reduction of the spectral weight of the ZR doublet is also seen in $\mathrm{NiO}$ when the $\mathrm{k}$ vector approaches the $\Gamma$ point. Here the same tight-binding argument applies: in the nearest 


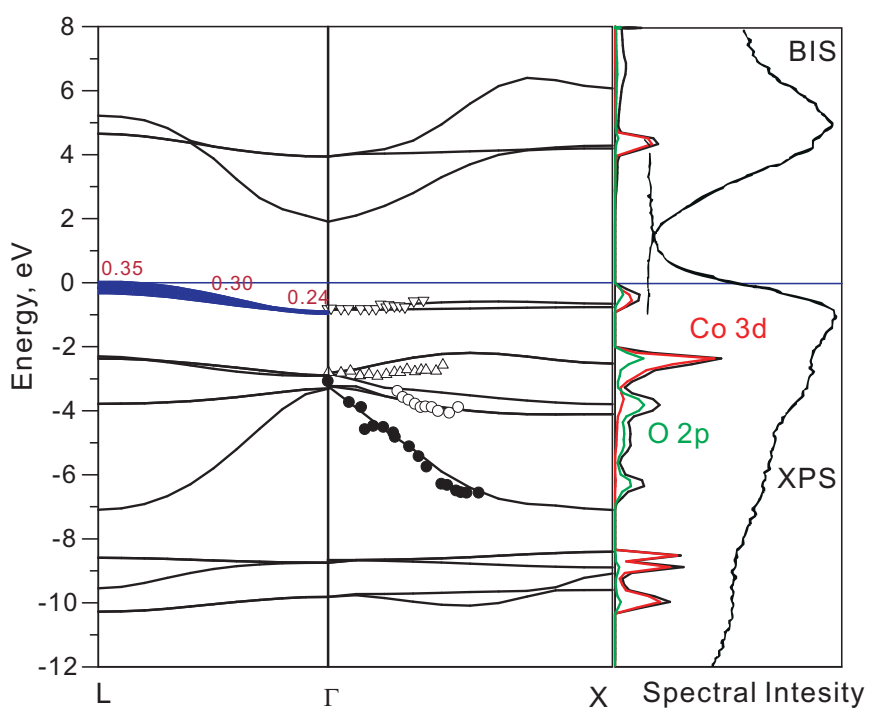

FIG. 3: (color online) The same as Fig. 2 but for CoO. Photoemission data are from Ref. [27, 28]

neighbor approximation the $\mathrm{k}$-dependent hybridization between oxygen $p$ and $d_{e_{g}}$ orbitals vanishes prohibiting the creation of the ZR state. The amount of actual $p$ electrons in the ZR state is shown by fattening this band in Fig. 2, While it decreases when $\mathbf{k} \rightarrow 0$, there is some residual amount around $\Gamma$ due to other hoppings which results in the actual number of electrons (shown by numbers) to be not as drastically reduced at $\Gamma$ as in the case of HTSCs.

Our result for $\mathrm{CoO}$ demonstrates very similar behavior to that of $\mathrm{NiO}$. Fig 3 compares the calculated quasiparticle dispersions with the photoemission data 27,28$]$. The calculated energy gap is $\sim 2.5 \mathrm{eV}$, and the experimental value is about $2.4 \mathrm{eV}$. Remarkably similar to $\mathrm{NiO}$, the $d$-electron spectral weight is distributed between the Hubbard bands found at energies $-9 \sim-10 \mathrm{eV}$ and at $4 \mathrm{eV}$, as well as the ZR band just below the Fermi level. The lowest excitation here can be described as a spin triplet state because of the antiferromagnetic interaction between the oxygen hole and the 3 hole $S=3 / 2$ state of the Co ion. We also see that the spectral weight in the ZR triplet band gets reduced as one moves towards the BZ center exactly as in the previous cases.

We finally mention our calculations for $\mathrm{FeO}$ and $\mathrm{MnO}$ where a very similar behavior was found. Since the spin momenta on Fe and $\mathrm{Mn}$ sites are roughly equal to 2 and $5 / 2$, respectively, the corresponding generalizations of the ZR states are spin 3/2 (quadruplet) for $\mathrm{FeO}$, and spin 2 (quintet) for $\mathrm{MnO}$. We should however mention that moving towards middle of the $3 d-$ metal oxide series, the hybridization effects become more and more pronounced and our calculations based on small cluster diagonalization may be less accurate.
In conclusion, we have performed cluster exact diagonalization based LDA+DMFT calculations for quasiparticle spectra in selected transition metal oxides. Low energy excitations were compared in details with the ARPES experiments including the ZR states in selected HTSCs, as well as doublet, triplet, quadruplet and quintet states in $\mathrm{NiO}, \mathrm{CoO}, \mathrm{FeO}, \mathrm{MnO}$, respectively. Generally good agreement between the theory and experiments was found using single site DMFT approximation for classical Mott-Hubbard systems, while for the case of the cuprates, going beyond the single impurity is essential. The reduction of the spectral weight in the ZR band when approaching the $\Gamma$ point was monitored for all materials and explained based on the tight-binding argument.

The authors acknowledge useful conversations with G. Kotliar and G. Sawatzky. The work was supported by NSF DMR Grants No. 0608283, No. 0606498. X.G.W. acknowledges support from National Key Project for Basic Researches of China (2006CB921802), and Natural Science Foundation of China under Grant No. 10774067.

[1] M. Imada, et.al, Rev. Mod. Phys. 70, 1039 (1998).

[2] W. Pickett, Rev. Mod. Phys. 61, (1989).

[3] A. Damascelli, et.al, Rev. Mod. Phys. 75, 473 (2003).

[4] K. Terakura, et.al., Phys. Rev. Lett. 52, 1830 (1984).

[5] Z.-X. Shen, et.al, Phys. Rev. B 44, 3604 (1991).

[6] Theory of the Inhomogeneous Electron Gas, ed. by S. Lundqvist et.al. (Plenum, NY, 1983).

[7] V. I. Anisimov, et.al, Phys. Rev. B 44, 943 (1991).

[8] J. Zaanen, et.al, Phys. Rev. Lett. 55, 418 (1985).

[9] F. C. Zhang et.al, Phys. Rev. B 37, 3725 (1988).

[10] G. Kotliar, et.al, Rev. Mod. Phys. 78, 865 (2006).

[11] A. Lichtenstein et.al, Phys. Rev. B 57, 6884 (1998).

[12] X. Wan, et.al, Phys. Rev. Lett. 97, 266403 (2006).

[13] X. Ren, et.al, Phys. Rev. B 74, 195114 (2006); J. Kuneš, et.al, Phys. Rev. B 75, 165115 (2007).

[14] F. Ronning et.al, Phys Rev. B 71, 094518 (2005); T. Valla et.al., Phys. Rev. Lett. 98, 167003 (2007); D. S. Inosov et.al. Phys. Rev. Lett. 99, 237002 (2007).

[15] J. Bała, et.al, Phys. Rev. Lett. 72, 2600 (1994).

[16] S. Y. Savrasov, et.al, Phys. Rev. Lett. 96, 036404 (2006).

[17] A. Weibe, et.al, Rev. Mod. Phys. 78, 275 (2006).

[18] E. Dagotto, et.al, Phys. Rev. Lett. 74, 310 (1994); Belinicher, et.al, Phys. Rev. B 54, 14914 (1996).

[19] B. O. Wells, et.al, Phys. Rev. Lett. 74, 964 (1995).

[20] S. LaRosa, et.al, Phys. Rev. B 56, R525 (1997).

[21] C. Kim, et.al, Phys. Rev. Lett. 80, 4245 (1998)

[22] T. Tohyama et.al, Super. Sci. Technol. 13, R17 (2000).

[23] A. Macridin et.al, Phys. Rev. Lett. 99, 237001 (2007).

[24] A. Fujimori, et.al, Phys. Rev. B 29, 5225 (1984).

[25] G. A. Sawatzky et.al, Phys. Rev. Lett. 53, 2339 (1984).

[26] T. M. Schuler et.al, Phys. Rev. B 71, 115113 (2005).

[27] Z.-X. Shen et.al, Phys. Rev. B 42, 1817 (1990).

[28] J. van Elp et.al, Phys. Rev. B 44, 6090 (1991). 